Journal of Patient-Centered

\title{
An Objective Structured Clinical Examination Case for Opioid Management: Standardized Patient Ratings of Communication Skills as a Predictor of Systems-Based Practice Scores
}

Heidi Kenaga

Tsveti Markova

R. Brent Stansfield

Sarwan Kumar

Pierre Morris

Follow this and additional works at: https://aah.org/jpcrr

Part of the Community Health and Preventive Medicine Commons, Educational Assessment, Evaluation, and Research Commons, Medical Education Commons, Mental and Social Health Commons, and the Primary Care Commons

\section{Recommended Citation}

Kenaga H, Markova T, Stansfield RB, Kumar S, Morris P. An objective structured clinical examination case for opioid management: standardized patient ratings of communication skills as a predictor of SystemsBased Practice scores. J Patient Cent Res Rev. 2021;8:261-6. doi: 10.17294/2330-0698.1800

Published quarterly by Midwest-based health system Advocate Aurora Health and indexed in PubMed Central, the Journal of Patient-Centered Research and Reviews (JPCRR) is an open access, peer-reviewed medical journal focused on disseminating scholarly works devoted to improving patient-centered care practices, health outcomes, and the patient experience. 


\title{
An Objective Structured Clinical Examination Case for Opioid Management: Standardized Patient Ratings of Communication Skills as a Predictor of Systems-Based Practice Scores
}

\author{
Heidi Kenaga, PhD, ${ }^{1}$ Tsveti Markova, MD, ${ }^{1,2}$ R. Brent Stansfield, PhD, ${ }^{1}$ Sarwan Kumar, MD,, 4 \\ Pierre Morris, MD2,5 \\ ${ }^{1}$ Office of Graduate Medical Education, Wayne State University School of Medicine, Detroit, MI; ${ }^{2}$ Department of Family \\ Medicine and Public Health Sciences, Wayne State University School of Medicine, Detroit, MI; ${ }^{3}$ nternal Medicine \\ Residency Program, Ascension Providence Rochester Hospital, Rochester, MI; ${ }^{4}$ Department of Internal Medicine, \\ Wayne State University School of Medicine, Detroit, Ml; ${ }^{5}$ Family Medicine \& Transitional Year Residency Programs, \\ Ascension Providence Rochester Hospital, Rochester, MI
}

\begin{abstract}
The Wayne State University Office of Graduate Medical Education (WSUGME) uses an objective structured clinical examination (OSCE) to assess its programs' contribution to enhancing residents' communication skills. In response to revisions in Michigan's opioid-prescribing mandates in 2017, WSUGME developed a pain management case in collaboration with faculty and the Wayne State University School of Medicine to educate residents about these mandates while gauging their skills in Systems-Based Practice (SBP), an Accreditation Council for Graduate Medical Education Core Competency. This study examined whether resident OSCE performance predicted year-end milestones scores in SBP1 (coordinates patient care within various health care delivery settings), SBP2 (works in interdisciplinary teams to enhance patient safety and improve patient care quality), and SBP3 (practices and advocates for cost-effective, responsible care).

Participants included two cohorts of first- (PRG-1) and second-year (PRG-2) residents in 6 programs: one cohort from academic year 2018-2019 $(n=33)$, the other from 2019-2020 $(n=37)$. Before the OSCE, WSUGME emailed residents the new state prescription requirements. During the simulated encounter, standardized patients rated residents on a validated communication instrument, and WSUGME conducted a linear regression of patient ratings on resident SBP milestone scores. The ratings of communication skills of PRG-1 residents did not predict any of the year-end SBP milestones. However, ratings of communication skills of PRG-2 residents predicted SBP1 and SBP2, though not SBP3, milestones. The OSCE opioid case proved to be a valid measure of PRG-2 residents' competence gained across the first year but was less meaningful when applied to PRG-1 residents. ( $\mathrm{J}$ Patient Cent Res Rev. 2021;8:261-266.)
\end{abstract}

Keywords opioid prescription; pain management; residency training; Systems-Based Practice; OSCE

$\mathrm{I}$ n 2017, the Michigan state legislature passed new laws to heighten awareness of the dangers of opioid abuse in the context of the prescriber-patient relationship. ${ }^{1}$ In that year, the state had one of the highest rates of opioid overdose fatalities and prescription rates nationally, with more than 21 deaths per 100,000 and 74 prescriptions per 100 patients. ${ }^{2}$ Studies have indicated

Corresponding author: Heidi Kenaga, PhD,

Wayne State University School of Medicine, Graduate Medical Education, 540 E. Canfield St., Scott Hall Room 4374, Detroit, MI 48201 (hkenaga@med.wayne.edu) the need for greater pain management training starting in the undergraduate curriculum, as only $16 \%$ of schools offer electives on this topic and even fewer focus on opioid prescription practices. ${ }^{3}$ While some government organizations have created clinical guidelines for the management of opioids, ${ }^{4}$ few residents have had formal training in employing these principles in clinical settings.

Since 2012, Wayne State University's Office of Graduate Medical Education (WSUGME) has required that all residents participate in an objective structured clinical examination (OSCE) during the second month of both their first (PRG-1) and second (PRG-2) program years as a means to assess their clinical communication skills. Data 
from this activity generate estimates of how much change can be expected and how much of that performance is attributable to the first year of training. WSUGME uses different standardized patient cases each year. In response to state changes in opioid prescribing practices, the office developed a pain management standardized patient case for the OSCE in 2018, drawing upon an existing model developed by Alford and colleagues. ${ }^{5}$ WSUGME sought to train residents in the new state guidelines, which mandate provider discussion of the risks of opioid prescription and alternative treatment options.

During each year of training, the clinical competency committees in all WSUGME programs use the Accreditation Council for Graduate Medical Education (ACGME) milestones as benchmarks for determining resident progress. The committees assess residents' skill level at the start of training and after 1 year by their facility in Systems-Based Practice (SBP), an ACGME competency that requires trainees to "demonstrate an awareness of and responsiveness to the larger context and system of health care and the ability to effectively call on system resources to provide care that is of optimal value." ${ }^{, 7} 7$ Medical educators have discussed the difficulty of determining SBP competencies in practical, empirical ways because the conceptual underpinnings of SBP are not easily measured in observable actions. ${ }^{8}$ Some researchers have argued that simulated experiences like the OSCE may be a powerful tool in assessing SBP competencies, ${ }^{9-11}$ and one of our study goals was to provide validity evidence for using this training activity to gauge residents' performance on the SBP milestone. The instrument used in our OSCE, the Kalamazoo Essential Elements Communication Checklist-Adapted (KEECC-A), contains two items (shares information, reaches agreement) that both align closely with the SBP core competencies and assess residents' understanding of state mandates designed to enhance pain management discussions with patients. ${ }^{12}$

The medical education literature contains noteworthy examples of pain management training models. Vettese et $\mathrm{al}^{13}$ reported the impact of a safe opioid-prescribing educational program on residents' knowledge, confidence, and self-reported practices. As part of the protocol, participants engaged in simulated techniques (role play) with each other following a didactic component that addressed principles of patient-centered communication. Other studies have reported on outcomes of simulated training experiences like the OSCE on the SBP and Practice and Improvement competencies. ${ }^{9,10}$ However, to the best of our knowledge, there are no studies detailing an opioid pain management OSCE case that employs a validated instrument for assessing trainee clinical communication skills, particularly with regard to determining gains in engaging in shared decision-making with patients regarding treatment options available in various health care delivery settings.

The study described herein relied on performance ratings provided by experienced standardized patients who completed a validated communication instrument after the simulated encounter. This instrument's items also are aligned with ACGME Core Competencies. Our study aimed to show how tools like the OSCE can provide educators with valuable data about trainees' knowledge gains that can transform pain management curricula and improve patient outcomes.

\section{METHODS}

For this study, WSUGME adopted a cross-specialty "harmonized" version of SBP, developed by Edgar and colleagues under the auspices of the ACGME, that was sufficiently general to encompass the core goals of this milestone across primary care and surgical specialties. ${ }^{7}, 14$ The milestone comprises 3 subcompetencies: SBP1 - coordinates patient care within various health care delivery settings, SBP2 - works in interdisciplinary teams to enhance patient safety and improve patient care quality, and SBP3 - practices and advocates for costeffective, responsible care. These subcompetencies were derived from a qualitative analysis of 4 milestones used in multiple specialties to determine common themes addressed by each. Pertinent SBP themes included navigation of multiple health care delivery settings, utilization of community resources, and understanding of health economics. ${ }^{14}$ In short, this milestone assesses a resident's expertise in systems thinking - how to optimize treatment in the context of patient needs, the care team, the clinical setting, and the policy landscape. ${ }^{7}$

WSUGME requires that all PRG-1 and PRG-2 residents in all specialties participate in OSCE training activities, which are held in the month following the program orientation. OSCEs in the Wayne State University School of Medicine are conducted by the Kado Clinical Skills Center, whose staff are trained to develop new cases to meet emergent challenges in the health care environment. In spring of 2018, WSUGME asked Kado staff to adapt a case addressing safe opioid prescribing practices. ${ }^{5}$ Kado sent drafts of the opioid case to the program directors of internal medicine and family medicine for review, and WSUGME conducted a pilot in the early summer attended by a faculty member, 2 family medicine residents, and 2 internal medicine residents, all of whom provided feedback incorporated into the revised version. In preparation for the OSCE training activity, Kado began training several standardized patients in the case in the late summer of 2018. 
The standardized patient used in this project was "Morgan Tempo," a 59-year-old woman with chronic lower back pain, the result of degenerative changes at multiple lumbar levels and mild spinal stenosis. Tempo has tried other pain-relief methods, but only Norco ${ }^{\circledR}$ (hydrocodone + acetaminophen) obtained from a neighbor eased her pain. She seeks a prescription since she must return to work as a teacher after being on medical leave for 2 months and is very reluctant to consider other methods of pain relief. The standardized patient's goal during this simulated patientprovider interaction is to assess the skill with which the resident is able to convey the benefits of and advocate for alternative treatment options to opioid use in order to ensure patient safety and optimize quality of care.

About 1 week prior to the OSCE administration in 2018 and 2019, WSUGME emailed participants all case notes and Michigan's Opioid Start Talking form, which mandates provider discussion of the risks of opioid prescription and addressing of nonopioid pain management strategies with patients. ${ }^{15}$ All WSUGME curricula include didactics on pain management, and at least 2 (anesthesiology and family medicine) had a quality improvement project related to this topic. However, only family medicine's project referred to the Opioid Start Talking form.

WSUGME staff and program faculty champions observed the live encounters via remote video system, and the Kado Clinical Skills Center taped each encounter for later analysis. At the conclusion of each encounter, standardized patients rated residents' performance using the KEECC-A. ${ }^{12}$ The KEECC-A was selected because it is better validated and the most widely used tool for the assessment of medical trainees' communication skills. In addition, its 7 items are aligned with ACGME milestones.

After the OSCE administration for that morning or afternoon was completed, participants engaged in a 15-minute debrief session with WSUGME staff and faculty champions. This offered residents an opportunity to reflect further on the simulation experience and receive immediate feedback on their performance. Subsequently, after Kado sent each participant a video of their simulated encounters, faculty provided individualized feedback together with WSUGME reports.

The university's institutional review board determined that this retrospective study did not involve human subjects research and thus was exempt from board review.

\section{RESULTS}

Participants included two cohorts of PRG-1 and PRG-2 residents from 6 programs (anesthesiology, dermatology, family medicine, internal medicine, otolaryngology, and urology). The number of possible participants was 36 in 2018-2019 and 38 in 2019-2020; the actual number of participants with usable data for each study year was 33 and 37 , resulting in participation rates of $92 \%$ and $97 \%$, respectively. In the 2018-2019 administration of the OSCE, the opioid case was new for all PRG-1 and PRG-2 residents. During the second administration in 2019-2020, all rising PRG-2s took the same case again. Participants with unusable data included 2 residents who transferred into the program in the second year and 2 residents who were unable to attend their first-year OSCE.

Following the end of the OSCE training, WSUGME conducted a linear regression analysis with standardized patients' KEECC-A scores as predictors and residents' SBP milestone ratings as outcomes. Patient ratings on the KEECC-A were found to be reliable (Cronbach's alpha: 0.85 ). Linear regression determined that the communication skills of PRG-1 residents did not predict year-end SBP milestones $\left(R^{2}:<0.01 ; \mathrm{P}>0.05\right)$. Communication skills of PRG-2 residents did predict SBP1 $\left(R^{2}: 0.39 ; \mathrm{P}<0.001\right.$; Figure 1) and SBP2 $\left(R^{2}: 0.32 ; \mathrm{P}<0.001\right.$; Figure 2$)$ but not SBP3 $\left(R^{2}:<0.01 ; \mathrm{P}>0.05\right.$; Figure 3$)$.

SBP1 and SBP2 ratings were highly correlated among PRG-1 residents ( $r=0.92)$. SBP3 was less correlated with these ( $r=0.79$ with SBP1 and $r=0.71$ with SBP2), suggesting that the meanings of these subcompetencies are interrelated and that SBP3 is more distinct. It is possible that SBP3's focus on cost-effectiveness (an aspect not contained in the other SBP subcompetencies) caused this distinctiveness.

\section{DISCUSSION}

This study provides medical educators with a practical tool for effectively predicting residents' SBP competency using a robust assessment protocol and a novel and timely OSCE case involving pain management and opioid prescription. Because first-year OSCE scores prior to the start of training were unrelated to first-year SBP subcompetency ratings, but second-year OSCE scores were linearly related, training in the first year of residency is associated with the acquisition of SBP-related skills that are observable in clinical settings (as measured by subcompetency ratings) and performance on the standardized patient case. The absence of the effect in the first year suggests the result is not due to halo effects or a resident's general skills in communication since those differences would have been present in the first-year data as well. That this relationship did not exist for PRG-1 residents regarding their future performance suggests that a level of SBP competence is gained during the first year of residency and that our OSCE pain management case is a valid measure of that competence. 

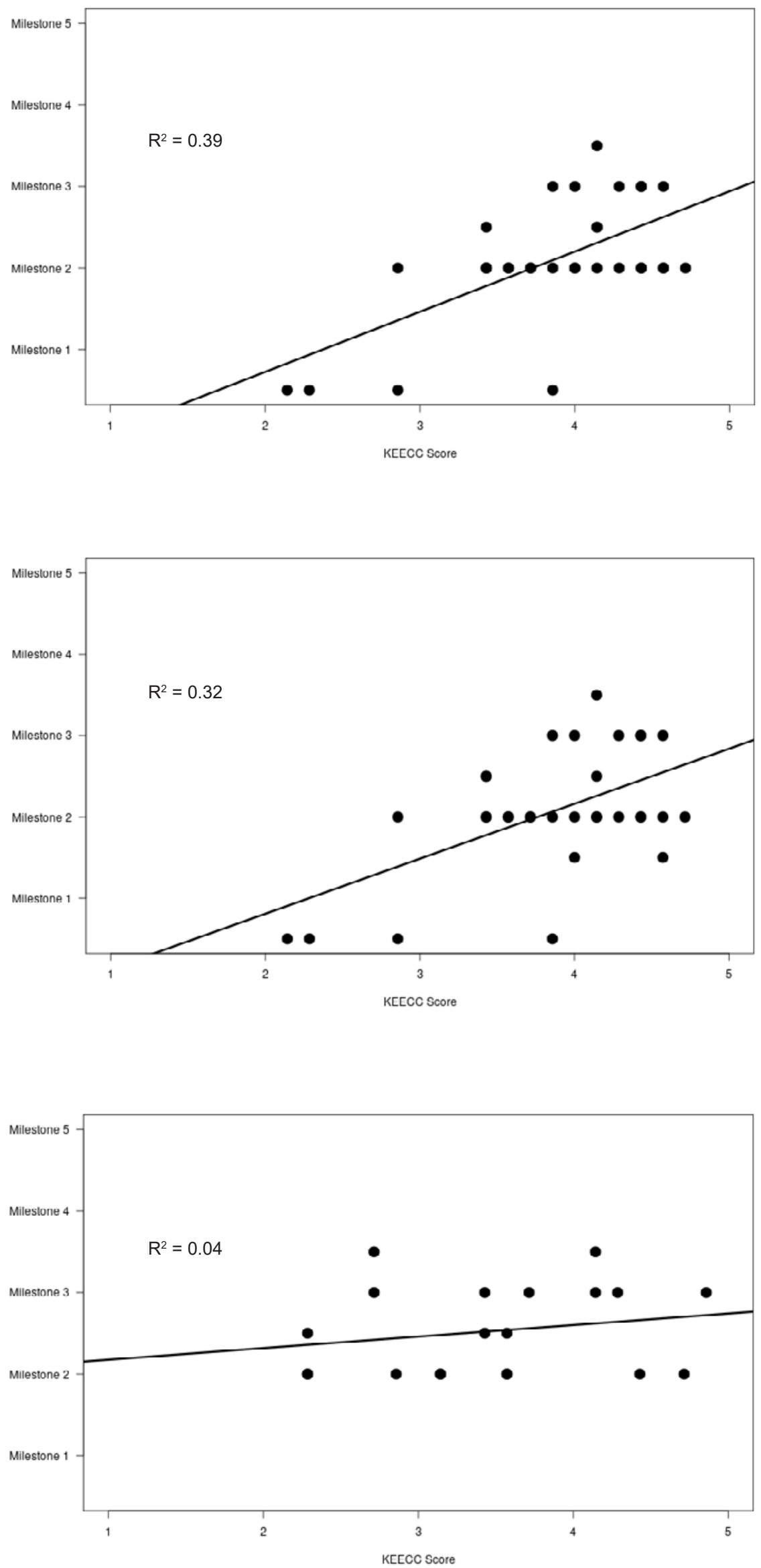

Figure 1. Milestone ratings for PRG-2 residents in SystemsBased Practice subcompetency 1 (ie, coordinates patient care within various health care delivery settings) as calculated by Kalamazoo Essential Elements Communication Checklist-Adapted (KEECC-A) mean scores.

Figure 2. Milestone ratings for PRG-2 residents in Systems-Based Practice subcompetency 2 (ie, works in interdisciplinary teams to enhance patient safety and improve patient care quality) as calculated by Kalamazoo Essential Elements Communication Checklist-Adapted (KEECC-A) mean scores.

Figure 3. Milestone ratings for PRG-2 residents in SystemsBased Practice subcompetency 3 (ie, practices and advocates for cost-effective, responsible care) as calculated by Kalamazoo Essential Elements Communication Checklist-Adapted (KEECC-A) mean scores. 
Our finding that PRG-2 residents' communication scores did not predict year-end assessments of SBP3 (ie, practices and advocates for cost-effective, responsible care) is likely the result of the goals of the case, which were less about cost and more about shared decisionmaking between provider and patient regarding the range of alternatives to opioid prescription. In the setting of pain management, the most effective care isn't always the least expensive.

We believe this OSCE case is particularly suitable for assessing ACGME's SBP competency and thus valuable to GME educators and program directors. It requires fairly complex communication behaviors demonstrating both awareness of and sensitivity to the patient's situation (inability to find pain relief while needing to return to work) while fulfilling regulatory requirements (jointly exploring available resources for alternative types of pain management while facing the patient's strong resistance to same). The case may function to identify residents with communication deficits as well as training gaps that might be addressed via additional interventions and revisions to curricula. ${ }^{9}$

The Michigan Department of Health and Human Services has reported that, following a period of decline, there have been alarming surges in the incidence of opioid overdoses during the COVID-19 pandemic (as of the spring of 2020). ${ }^{16}$ As such, the inclusion in medical education of evidence-based nonpharmacological methods of pain management and alternatives to opioid prescription remains a pressing issue.

Limitations of this study include a small sample size, a sample from one institution only, and use of a fairly new standardized patient case. In addition, the use of experienced standardized patients entails significant cost, although this is likely the most effective way to assess and enhance resident communication skills in a controlled setting. WSUGME will repeat the analysis after conducting the opioid pain management case as a virtual OSCE training exercise with our 2020-2021 PRG-1 and PRG-2 residents. Future research could address the reliability of the observed effects on subsequent cohorts and the validity of simulated telemedicine encounters for SBP and other ACGME Core Competencies.

\section{CONCLUSIONS}

The current opioid epidemic has highlighted the need not just for transformations in prescribing practices but also better tools for improving training protocols so that residents are best equipped to provide optimal care for patients seeking relief from pain. Given the challenge of evaluating Systems-Based Practice from observed behaviors in actual clinical environments, our findings suggest that an objective structured clinical examination case targeting an important public health concern can provide useful data for such an assessment.

\section{Patient-Friendly Recap}

- New physicians often have little formal training in opioid prescription practices. The increase in opioid overdose fatalities in Michigan has highlighted the need for better pain management education so that doctors are best equipped to care for patients seeking pain relief and can provide alternative treatment options available to them.

- Graduate medical educators in Detroit, Michigan, used standardized patients trained in providing simulated clinical experiences in an effort to teach resident physicians how to better communicate safe pain management practices.

- In this study of a clinical simulation exercise, second-year residents showed marked improvement in communicating alternatives to opioid prescription.

\section{Acknowledgments}

The authors thank the Kado Clinical Skills Center at the Wayne State University (WSU) School of Medicine for their administration of the standardized patient case to WSU residents and their collaboration with WSU Office of Graduate Medical Education staff in providing outcomes. Special thanks are due to Kado staff member Sonal Patel for her work on adapting the pain management case.

\section{Author Contributions}

Study design: Kenaga, Markova, Stansfield. Data acquisition or analysis: all authors. Manuscript drafting: Kenaga, Markova, Stansfield. Critical revision: all authors.

\section{Conflicts of Interest}

None.

\section{References}

1. Department of Licensing and Regulatory Affairs, Michigan Department of Health and Human Services. Michigan opioid laws: frequently asked questions (FAQs). Document updated March 6, 2019; accessed September 6, 2020. https://www. michigan.gov/documents/lara/LARA DHHS Opioid Laws FAQ_05-02-2018_622175_7.pdf

2. National Institute on Drug Abuse. Opioid summaries by state. Page updated April 16, 2020; accessed September 6, 2020. https://www.drugabuse.gov/drugs-abuse/opioids/opioidsummaries-by-state

3. Mezei L, Murinson BB; Johns Hopkins Pain Curriculum Development Team. Pain education in North American medical schools. J Pain. 2011;12:1199-208. $\underline{\text { CrossRef }}$ 
4. U.S. Department of Veterans Affairs. VA/DoD clinical practice guidelines. Management of opioid therapy (OT) for chronic pain (2017). Accessed December 21, 2020. https://www. healthquality.va.gov/guidelines/Pain/cot/

5. Alford DP, Carney BL, Brett B, Parish SJ, Jackson AH. Improving residents' safe opioid prescribing for chronic pain using an objective structured clinical examination. J Grad Med Educ. 2016;8:390-7.

6. Dyne PL, Strauss RW, Rinnert S. Systems-based practice: the sixth core competency. Acad Emerg Med. 2002;9:1270-7.

7. Edgar L, Roberts S, Holmboe E. Milestones 2.0: a step forward. J Grad Med Educ. 2018;10:367-9. CrossRef

8. Chen EH, O'Sullivan PS, Pfennig CL, Leone K, Kessler CS. Assessing systems-based practice. Acad Emerg Med. 2012;19:1366-71. CrossRef

9. Varkey P, Natt N, Lesnick T, Downing S, Yudkowsky R. Validity evidence for an OSCE to assess competency in systems-based practice and practice-based learning and improvement: a preliminary investigation. Acad Med. 2008;83:775-80. CrossRef

10. Heirich MS, Sinjary LS, Ziadni MS, et al. Use of immersive learning and simulation techniques to teach and research opioid prescribing practices. Pain Med. 2019;20:456-63. CrossRef

11. Hoelzer BC, Moeschler SM, Seamans DP. Using simulation and standardized patients to teach vital skills to pain medicine fellows. Pain Med. 2015;16:680-91. CrossRef
12. Porcerelli JH, Brennan S, Carty J, Ziadni M, Markova T. Resident ratings of communication skills using the Kalamazoo Adapted Checklist. J Grad Med Educ. 2015;7:458-61. CrossRef 13. Vettese TE, Thati N, Roxas R. Effective chronic pain management and responsible opioid prescribing: aligning a resident workshop to a protocol for improved outcomes. MedEdPORTAL. 2018;14:10756. CrossRef

14. Edgar L, Roberts S, Yaghmour NA, et al. Competency crosswalk: a multispecialty review of the Accreditation Council for Graduate Medical Education milestones across four competency domains. Acad Med. 2018;93:1035-41. CrossRef

15. Michigan Department of Health and Human Services. Opioid Start Talking form. Document revised March 2020; accessed September 6, 2020. https://www.michigan.gov/documents/ mdhhs/MDHHS-5730 $621248 \quad 7 . d 0 t$

16. Michigan Department of Health and Human Services. Opioid overdoses surge during COVID-19 pandemic; MDHHS promotes treatment and resources. News release issued July 29, 2020; accessed September 6, 2020. https://www.michigan. gov/som/0,4669,7-192-29942_34762-535078--,00.html

(C) 2021 Advocate Aurora Health, Inc. 